\title{
Automatic beam alignment for the mode-cleaner cavities of GEO 600
}

\author{
Hartmut Grote, Gerhard Heinzel, Andreas Freise, Stefan Goßler, Benno Willke, \\ Harald Lück, Harry Ward, Morag M. Casey, Kenneth A. Strain, David Robertson, \\ Jim Hough, and Karsten Danzmann
}

\begin{abstract}
The automatic alignment system for the two suspended, triangular mode-cleaner cavities of the gravitational wave detector GEO 600 has been in continuous operation since the spring of 2001. A total of 20 angular degrees of freedom are controlled by feedback loops with almost no manual alignment having been required since installation. Error signals for the eight most important degrees of freedom are obtained with the differential wave-front sensing technique. We describe the sensing system, the control topology, and the performance of the automatic alignment system. A continuous locking stretch of more than $120 \mathrm{~h}$ has been achieved. (C) 2004 Optical Society of America

OCIS codes: $120.3180,230.0040$.
\end{abstract}

\section{Introduction}

Laser interferometric gravitational wave detectors are the most precise interferometers ever built. To allow for outstanding displacement sensitivities, the mirrors need to be isolated from seismic motion. For this purpose the cavity mirrors and beam-steering mirrors are suspended as pendulums inside a vacuum system. Although this gives good attenuation of longitudinal and angular motions of the suspended components in the measurement band of a gravitational wave detector (e.g., $50 \mathrm{~Hz}-1 \mathrm{kHz}$ ), the motion is enhanced at the pendulum resonant frequencies (typically around $1 \mathrm{~Hz}$ ). Moreover, suspended components are subject to larger drifts over long time periods than they would if rigidly mounted.

For achieving stable operation in the long term with suspended optical cavities, an automatic angu-

H. Grote (hrg@mpq.mpg.de), A. Freise, and S. Goßler are with the Institut für Atom- und Molekülphysik, Universität Hannover, Callinstrasse 38, D-30167 Hannover, Germany. G. Heinzel is with the Max-Planck-Institut für Gravitationsphysik, Teilinstitut Hannover, Callinstrasse 38, D-30167 Hannover, Germany. B. Willke, H. Lück, and K. Danzmann are with the Institut Für Atomund Molekülphysik and the Max-Planek-Institut für Gravitationsphysik. H. Ward, M. M. Casey, K. A. Strain, D. Robertson, and J. Hough are with Department of Physics and Astronomy, University of Glasgow G128QQ, Scotland, U.K.

Received 8 December 2003; revised manuscript received 8 December 2003; accepted 17 December 2003.

0003-6935/04/091938-08 $\$ 15.00 / 0$

(C) 2004 Optical Society of America lar alignment system is required for two degrees of freedom of each suspended mirror.

This paper describes the automatic alignment system implemented for the two suspended modecleaner cavities of GEO 600. ${ }^{1}$ Mode cleaners are used to suppress higher-order TEM modes of the laser beam used for the main length-sensing interferometer of a gravitational wave detector. In addition, they provide temporal filtering of amplitude and frequency fluctuations.

Each of the GEO 600 mode cleaners consists of three mirrors forming a triangular optical cavity. Together with two beam-steering mirrors for each mode cleaner, a total of ten suspended mirrors, and thus 20 angular degrees of freedom, has to be aligned. Vertical pointing of a laser beam is referred to as the pitch and horizontal pointing as the yaw motion of an associated mirror. Roll motion around the optical axis has no effect on path length and alignment.

The goal of the automatic alignment system is to keep the fundamental optical eigenmode of each cavity colinear with the incoming beam and to center all beam spots on the corresponding mirrors. Both of these tasks have to be performed reliably over long time periods, with as little human interaction as possible to permit continuous long-term operation of a gravitational wave observatory.

Aligning the cavity axis with the incoming beam is the most important alignment task in that this yields maximized power transmission and minimizes the coupling of incident laser beam geometry fluctuations into the transmitted light power. It is necessary to 

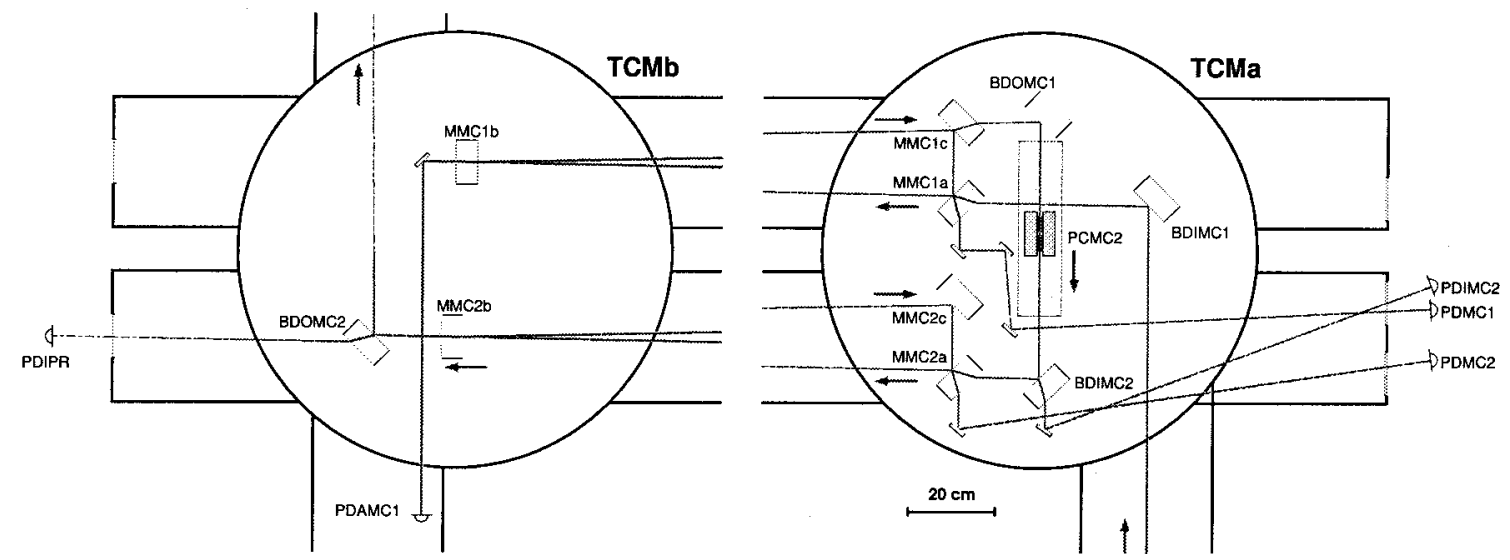

Fig. 1. Optical setup of the two GEO 600 mode-cleaner cavities within the vacuum system. Arrows denote directions of light propagation. All photodetectors (including PDAMC1) are located outside the vacuum envelope. The distance between the two vacuum chambers is $4 \mathrm{~m}$, resulting in a round-trip length of each of the two mode cleaners of approximately $8 \mathrm{~m}$. The first triangular mode cleaner is formed by MMC1a, MMC1b, and MMC1c and the second by MMC2a, MMC2b, and MMC2c.

produce the required alignment error signals, which yield information about the four degrees of freedom (two angles and two lateral displacements) describing the relative orientation of a cavity axis to the corresponding input beam. To obtain such signals, we use the differential wave-front sensing (DWS) technique (see, e.g., Refs. 2-4). It is an extension of the widely known Pound-Drever-Hall sensing scheme, ${ }^{5}$ which is used in separate control loops to keep the two GEO 600 mode cleaners on resonance with the incident laser light.

In the Pound-Drever-Hall scheme the phase difference between the interfering wave fronts of the cavity input beam and the beam leaking out of the cavity is averaged over the whole cross section of these two interfering beams. The resulting signal is fed to an appropriate feedback loop to keep the incident light in resonance with the cavity. That this loop is working correctly is an assumption throughout this paper.

The DWS technique uses split photodiodes to determine the angles between the two interfering wave fronts by subtraction of the phase difference of the interfering beams on the appropriate photodiode segments. If two such photodiodes are used (located near and far from the beam waist), the four measured angles between the wave fronts can be translated into angular and parallel displacements of the cavity axis with respect to the axis of the incoming beam.

The theory for obtaining alignment error signals with the DWS technique has been published already (see, e.g., Ref. 2), and the alignment signals for the GEO 600 mode cleaners have also been calculated. ${ }^{6}$

After giving a short overview of the optical setup of the mode cleaners (Section 2), we describe the experimental setup of the alignment system (Section 3), the alignment-control loop design, and the performance (Section 4) of the two mode cleaners of GEO 600. An important new (to our knowledge) result of this research is the successful long-term operation and excellent robustness achieved with the described system.

\section{Optical Setup of the GEO 600 Mode Cleaners}

Figure 1 shows the optical setup of the two modecleaner cavities of GEO 600 within the vacuum system. The mode-cleaner cavities and suspensions are described in detail in a companion paper. ${ }^{7}$

Light from the laser bench enters the vacuum chamber TCMa from the lower right, is directed by a beam-steering mirror (BDIMC1), and enters the first mode cleaner (MC1), consisting of two flat mirrors (MMC1a and MMC1c) and a curved mirror (MMC1b). The light leaves MC1 via MMC1c and is steered by mirror BDOMC1. Phase modulation is applied by a suspended electro-optic modulator (PCMC2). Another suspended mirror (BDIMC2) steers the beam toward the second mode cleaner (MC2), consisting of two flat mirrors (MMC2a and MMC2c) and a curved mirror (MMC2b). The light leaves MC2 via MMC2b and is finally directed toward the main interferometer by mirror BDOMC2 in chamber TCMb.

All beams required for the alignment system are drawn, together with corresponding photodetectors. The photodetectors PDAMC1, PDIMC2, and PDIPR are single (four-element) spot-position-detecting photodiodes, whereas PDMC1 and PDMC2 symbolize more complex arrangements, each using two (fourelement) photodiodes.

The detection setup PDMC1 and PDMC2 provide the four DWS signals needed for each mode cleaner. Error signals for the remaining 12 angular degrees of freedom of the ten suspended mirrors are taken from the beam spot positions on all five detectors shown and an additional spot-position sensor (not shown in Fig. 1), detecting the position of the beam steered by BDOMC2. Spot-position information is needed to center a beam on its respective mirror. This is especially important for cavity mirrors, in order to min- 


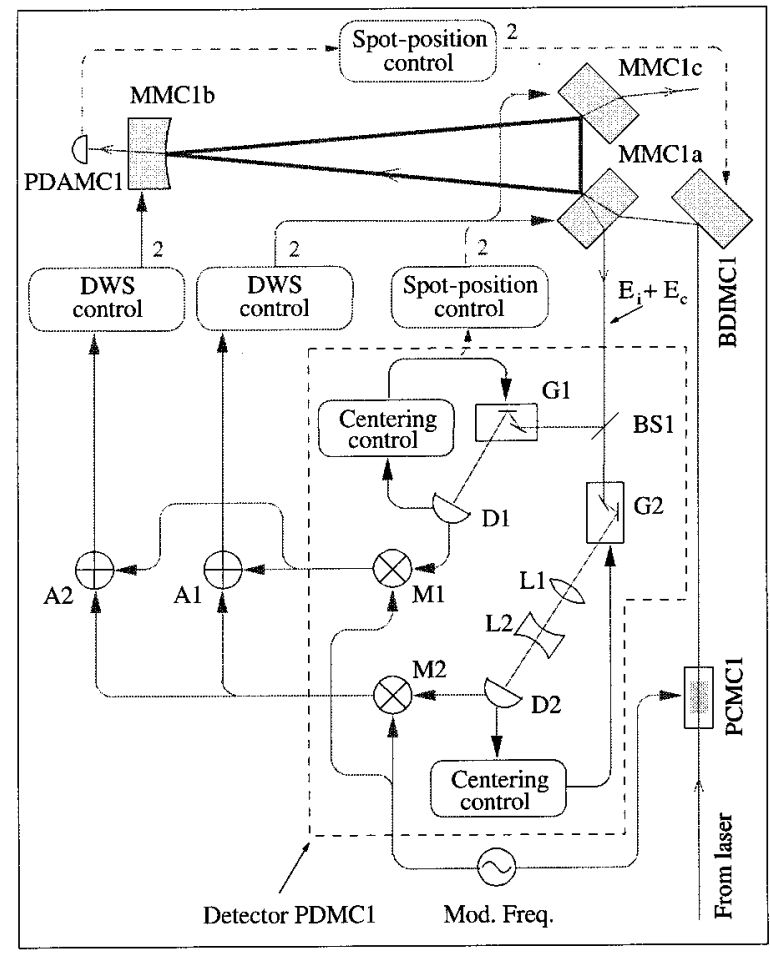

Fig. 2. Automatic alignment system layout for one mode cleaner. Details of the purpose of lenses L1 and L2, galvanometer scanners G1 and G2, photodiodes D1 and D2, mixers M1 and M2, and adders $\mathrm{A} 1$ and $\mathrm{A} 2$ are given in the text. The numbers at the control boxes denote the number of degrees of freedom controlled by each path.

imize coupling of yaw and pitch noise of mirrors into length changes of the optical cavity.

\section{Automatic Alignment Setup}

\section{A. Overview}

Figure 2 shows the most important part of the experimental setup for the automatic alignment system of MC1. To allow the use of the Pound-Drever-Hall locking and DWS, we have had the laser beam incident on MMC1a modulated in phase at $25.2 \mathrm{MHz}$ by using the electro-optic modulator PCMC1. The phase-modulation frequency is chosen to ensure that the resulting sidebands are far from resonant in the cavity. (The free spectral ranges are $37.48 \mathrm{MHz}$ for $\mathrm{MC} 1$ and 37.16 $\mathrm{MHz}$ for $\mathrm{MC2}$ ).

The beam leaving mirror MMC1a downward in Fig. 2 consists of the interfering fields of the directly reflected part of the input beam $\left(E_{i}\right)$ and the field leaking out of the cavity $\left(E_{c}\right)$ and is denoted $E_{i}+E_{c}$. This beam is split by the beam splitter BS1 (which has a transmission of approximately $50 \%$ ).

The components G1 and G2 (described in Subsection 3.B) are beam-deflecting devices used to center the beams on photodetectors D1 and D2. The corresponding control loops are called the centering control.

Lenses L1 and L2 in the light path to D2 are used to project beam $E_{i}+E_{c}$ into the far field (see Subsec- tion 3.D). Each of the detectors D1 and D2 (described in more detail in Subsection 3.C) consists of a quadrant photodiode with associated electronics to yield information about the beam spot position, whereas the angle between the interfering wave fronts (the DWS signal) is available after the coherent demodulation performed by mixers $\mathrm{M} 1$ and M2. Appropriate linear combinations of the DWS signals are then processed by analog electronic circuits A1 and A2 to generate feedback signals to individual mirrors. (See Subsection 3.D for a description of this orthogonalization process.) Amplified with an appropriate gain and frequency response, these signals are fed back to mirror MMC1b and mirrors MMC1a - MMC1c, where MMC1a - MMC1c denotes a differential mode motion of this pair of mirrors. These loops are referred to as DWS control. Owing to the cavity geometry, the DWS system cannot distinguish between misalignments of mirror MMC1a and mirror MMC1c. Consequently, the same feedback is applied to both mirrors with the appropriate sign for each mirror. We call the resulting motion a differential mode motion of these two mirrors. Note that the relative signs of the differential modes are different for yaw and pitch motion: In a differential yaw motion the two mirrors are rotated in different directions, whereas a differential pitch mode is performed by a pitch of both mirrors in the same direction.

The dashed lines denote slow digital feedback loops used for spot-position control. The beam is centered on MMC1b by feeding the spot-position signal from PDAMC1 back to mirror BDIMC1.

There remains two more degrees of freedom of the three cavity mirrors to be controlled. These are the two common modes of mirrors MMC1a and MMC1c, denoted by MMC1a + MMC1c. Variations of MMC1a + MMC1c show up in the direction of beam $E_{i}+E_{c}$. As this beam is centered on D1 by G1 with high bandwidth, the information of the direction of beam $E_{i}+E_{c}$ is contained in the feedback to G1. This signal is fed back to MMC1a + MMC1c by another spot-position control.

The alignment setup for the second GEO 600 mode cleaner is identical, by use of a phase-modulation frequency of $13 \mathrm{MHz}$, which is applied by PCMC2 in Fig. 1.

\section{B. Beam-Centering Scanners}

Experience on the Garching $30-\mathrm{m}$ prototype ${ }^{4}$ showed that it is useful to center the beam $E_{i}+E_{c}$ on the detecting quadrant photodiodes with auxiliary control loops. This permits easier lock acquisition of the alignment system and compensates for drifts of the detected beam with respect to the photodetector. In the case of three-mirror ring cavities there is an additional potential problem: The cavity beam can be perfectly aligned to the incoming beam but without enforcing a unique direction of the beam $E_{i}+E_{c}$. If beam $E_{i}+E_{c}$ is not centered on the quadrant diodes, the DWS signal size decreases and can contain offsets 


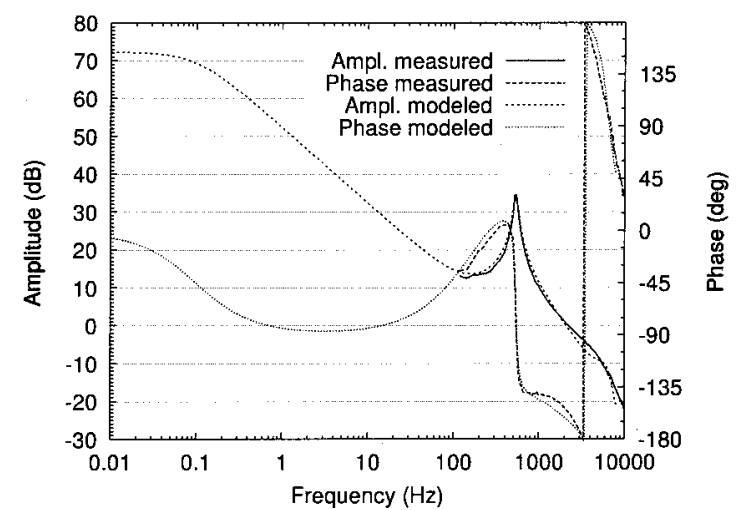

Fig. 3. Open loop gain and phase of the beam-centering loops. The resonance around $500 \mathrm{~Hz}$ is due to the scanners' mechanical resonance. These centering loops increase the dynamic range of the alignment system by more than an order of magnitude.

caused by higher transverse modes (e.g., $\mathrm{TEM}_{20}$ ). If, for example, beam $E_{i}+E_{c}$ deviates from the center of a diode by one beam radius, $86 \%\left(1-\mathrm{e}^{-2}\right)$ of the DWS signal is lost. If the centering scanners are not used, an angular pointing error of beam $E_{i}+E_{c}$ of roughly $200 \mu \mathrm{rad}$ leads to such a decentering on detector D2 in Fig. 2. By use of the scanners, beam $E_{i}+E_{c}$ can be moved up to $\pm 2000 \mu \mathrm{rad}$ while the beam is still well centered on D2, yielding the correct DWS signal. When the beam-pointing error exceeds $2000 \mu \mathrm{rad}$, the beam gets cut at the edges of the scanner mirrors. These numbers show that the dynamic range of the alignment-detection system is increased by more than a factor of 10 owing to the scanners. Such a large range allows the successful acquisition of the automatic alignment system even in highly misaligned initial states. This is demonstrated by Fig. 5 in Subsection 4.B.

G1 and G2 in Fig. 2 consist of two commercial galvanometer scanners (General Scanning Inc., Type G102), each carrying small $(10 \mathrm{~mm} \times 15 \mathrm{~mm})$ mirrors on their axes that can be rotated by \pm 2 deg. With perpendicular mounting of two scanners, one pair can point the reflected beam in two degrees of freedom. The centering loops in Fig. 2 control the spot position on the respective diodes with a bandwidth of around $1 \mathrm{kHz}$. The gain of this analog feedback loop is continuously normalized for the light power available on D1 and D2 by analog division of the position error signals. The centering loops for D1 and D2 are automatically switched off while the detected light power exceeds an upper or lower threshold. (For example, the scanners of the second mode cleaner are switched off if the first mode cleaner is not in lock, and thus no light is incident on the second mode cleaner.)

Figure 3 shows the open loop gain and phase of the centering loops. The gain peak around $500 \mathrm{~Hz}$ is caused by the scanners' main mechanical resonance. The suppression of beam-pointing errors on the photodetectors is $\approx 50 \mathrm{~dB}$ at the pendulum resonances around $1 \mathrm{~Hz}$.

\section{Photodetector Design}

The four quadrant photodetectors of PDMC1 and PDMC2 were built to the same design. We use Centronics QD50-3T quadrant photodiodes with a resonant electronic circuit for each of their quadrants, tuned to the modulation frequency of the detected beam $(25.2$ or $13 \mathrm{MHz})$. The resonant circuit rejects disturbances at frequencies away from the modulation frequency.

Typically in these applications a reverse voltage $U_{\text {bias }}$ is applied to the diode to reduce the junction capacity $C$ and increase the slew rate and bandwidth. If the slew rate and bandwidth are not critical, the choice of $U_{\text {bias }}$ can be guided by considerations of signal-to-noise performance and electrical dissipation. Decreasing $C$ (by increasing $U_{\text {bias }}$ ) increases the impedance of the resonant circuit $Z_{\max }$ on resonance, according to $Z_{\max }=L / R C$, where $L$ is the tunable inductance of the resonant circuit and $R$ is the series loss resistor of a photodiode quadrant. As the resonance frequency $\omega_{m}=1 / \sqrt{L C}$ is fixed, we get $Z_{\max }=1 / \omega^{2}{ }_{m} R C^{2}$.

Whereas the signal voltage across this impedance (being produced by the signal current) is proportional to $Z_{\text {max }}$, the thermal noise of the real part of $Z_{\max }$ is proportional only to $\sqrt{Z_{\text {max }}}$; thus the signal-to(thermal electronic) noise ratio increases proportional to $\sqrt{Z_{\max }}$, i.e., proportional to $1 / C$. Increasing $Z_{\text {max }}$ by increasing $U_{\text {bias }}$ is useful only until the photoelectron shot noise of the detected light gets larger than the thermal noise of $Z_{\max }$. Once the noise is dominated by shot noise only increasing the detected light power will increase the signal-to-noise ratio. On the other hand, upper limits to the detectable light power are given by the direct power dissipation of the absorbed light power plus the electrically dissipated power, which, in turn, depends on $U_{\text {bias }}$, with $P_{\text {el }}=U_{\text {bias }} * I_{\text {photo }} \quad$ (A current-limiting circuit prevents the photodiode from overheating owing to false operating conditions that expose the diode to too much light power.)

In most cases a lower limit to $U_{\text {bias }}$ will be set by the requirements of the slew rate and bandwidth. To handle the required bandwidth even at large signal amplitudes, we chose $U_{\text {bias }}=30 \mathrm{~V}$ for the $25.2-\mathrm{MHz}$ detector and $15 \mathrm{~V}$ for the $13-\mathrm{MHz}$ detector. Approximately $70 \mu \mathrm{A}$ of photocurrent per quadrant are required to have equal thermal electronic noise and photocurrent shot noise. The maximum possible photocurrent is, e.g., $2 \mathrm{~mA}$ per quadrant for $U_{\text {bias }}=$ $30 \mathrm{~V}$.

The resonant circuit of each quadrant is followed by a double-balanced mixer (Mini-Circuits TUF-3H with a $17-\mathrm{dBm}$ local oscillator and maximum $14-\mathrm{dBm}$ signal input). This demodulates the signal coherently with the given radio frequency. After demodulation and low-pass filtering, the sum and differences of all four quadrant signals are processed to give information about the angles between the detected wave fronts in two degrees of freedom.

In addition, the sum of the demodulated signals 
from the four quadrants is used for the longitudinal lock of the corresponding cavity. Furthermore, signals for the spot position of the beam on the quadrant photodiode are produced (without demodulation) and used as error signals for the beam-centering scanners. Finally, the total detected power (sum of all photocurrents without demodulation) is made available for normalization and monitoring purposes.

\section{Orthogonalization Setup}

The DWS signals generated from photodetectors D1 and D2 contain a linear combination of the alignment information, which therefore is (in the general case) not suitable for direct feedback to single mirrors.

Depending on the cavity geometry, individual mirror misalignments show up as a fixed combination of lateral and angular misalignments at the location of the beam waist [which is located halfway between the flat cavity mirrors MMC1(2)a and MMC1(2)c of the GEO mode cleaners]. With the propagation of beam $E_{i}+E_{c}$ away from the waist, a distance-dependent Gouy phase shift between the $\mathrm{TEM}_{00}$ and the $\mathrm{TEM}_{10 / 01}$ modes of $E_{i}+E_{c}$ is introduced. This phase shift determines which linear combination of lateral and angular misalignments a DWS sensor is most sensitive to. Hence the distribution of information on $\mathrm{D} 1$ and $\mathrm{D} 2$ depends on the distances of D1 and D2 from the waist. Obviously, D1 and D2 have to be placed at different distances to be sensitive to different linear combinations of misalignments. If the Gouy phase shift of the $\mathrm{TEM}_{00}$ and $\mathrm{TEM}_{10 / 01}$ modes at D1 and D2 differs by $90^{\circ}$, the sensing coordinate system is orthogonal, yielding the maximum possible signal levels.

The lens system consisting of L1 and L2 in Fig. 2 is used to adjust the desired Gouy phase shift on D2. The computation of the lens system follows the procedure described in earlier papers. ${ }^{4,6}$ The parameters of L1 and L2 are chosen to yield an additional Gouy phase shift of $90^{\circ}$ between the TEM ${ }_{00}$ and the $\mathrm{TEM}_{10 / 01}$ modes on D2 (compared with D1) and a reasonable spot size of approximately $3-\mathrm{mm}$ diameter on D2.

The next step of orthogonalization is the processing of the linear combinations of the DWS signals from D1 and D2 to match the set of chosen feedback points, which are the mirrors MMC1b and the combination of MMC1a + MMC1c, shown in Fig. 2.

These linear combinations are processed by use of an analog electronic matrix that is adjusted by the following procedure. An alignment dithering signal at a fixed frequency is temporarily applied to mirror $\mathrm{MMC} 1 \mathrm{~b}$, and the corresponding signal levels from D1 and D2 are measured. Then an appropriate linear combination of D1 and D2 signals is adjusted such that the resulting signal is close to zero at the dithering frequency. This signal can then be used for the feedback to mirrors MMC1a + MMC1c. In the same way, another linear combination of D1 and D2 is chosen to generate a feedback signal for MMC1b, which is zero if mirrors MMC1a + MMC1c are dithered. Note that, owing to the features of a triangu- lar cavity, the linear combinations of D1 and D2 required for horizontal alignment are different from those for vertical alignment.

The orthogonalization achieved in this way is better than 1:10, meaning that dithering of MMC1b shows up with less than $10 \%$ in the signal for MMC1a + MMC1c and vice versa.

\section{E. Servo Topology}

To superimpose the eigenmode axis of the mode cleaner onto the axis of the incoming beam, we feed the DWS signals back to the angular degrees of freedom of the cavity mirrors. The opposite approach of actuating on the pointing and displacement of the incident beam to match the actual cavity axis would also be possible with appropriate actuators. The choice of which alternative to use depends on which of the two reference frames (the suspended first mode cleaner or the laser) is quieter with respect to a frame in which the beam is to be used (the power-recycling cavity in case of GEO 600).

Measurements show that the beam jitter of the incident light on BDIMC1 in Fig. 2 is of the same order of magnitude as the residual motion of the mode-cleaner axis for frequencies up to a few hertz, which dominate the rms motion. Thus the difference between the alternatives appears insignificant in case of the first GEO 600 mode cleaner. For simplicity, the cavity mirrors were chosen as actuators. For the second mode cleaner there is no choice, as the only possible alignment actuators are the suspended mirrors.

The hierarchy of feedback systems for the modecleaner automatic alignment, as displayed in Fig. 2, is as follows:

- The beam-centering loops have the highest bandwidth (around $1 \mathrm{kHz}$ ), thus ensuring correct DWS signals whenever the mode-cleaner longitudinal control loops are closed.

- Once the mode cleaners are locked longitudinally, the feedback of the DWS signals via the DWS control loop to the cavity mirrors is switched on. These loops have a bandwidth of either 0.2 or $6 \mathrm{~Hz}$, depending on the type of feedback filter chosen (see Subsection 4.A).

- The lowest bandwidth (below $0.1 \mathrm{~Hz}$ ) is attained by the digital spot-position loops. The feedback loop centering the spot on mirror MMC1b in Fig. 2 by controlling alignment of BDIMC1 can be switched on only if the DWS control loop is working, so that the cavity axis follows the beam incident from BDIMC1.

Table 1 summarizes some properties of the three feedback loop types.

The analog electronic circuits of the DWS and centering control loops are digitally supervised; the servo systems can be switched on and off, and loop gains and offsets can be adjusted from a computer control system based on Labview. ${ }^{8}$ This computer control system guides the lock acquisition and automates the 
Table 1. Properties of the Three Feedback Loop Types of the Automatic Alignment System

\begin{tabular}{llcll}
\hline Feedback Loop Type & \multicolumn{1}{c}{ Sensor } & Loop Filter & Actuator & Bandwidth \\
\hline Centering control & Spot position on D1 and D2 & Analog & Scanner & $1 \mathrm{kHz}$ \\
DWS control & DWS signal & Analog & Cavity mirrors & 0.2 or $6 \mathrm{~Hz}$ \\
Spot-position control & Spot-position on QD & Digital & Steering mirror & $<0.1 \mathrm{~Hz}$ \\
\hline
\end{tabular}

${ }^{a}$ Four-quadrant detector for beam spot position sensing.

alignment control by means of subroutines called virtual instruments. The digital spot-position control is implemented by virtual instruments as well, running with time steps of $50 \mathrm{~ms}$.

Finally, another Labview-based digital loop ensures well-aligned cavities after a loss of lock. This offset shifting loop senses the analog feedbacks of the DWS loops and permanently adds digitally controlled offset voltages to these feedbacks, so that the dccomponents of these analog feedback signals are minimized. The alignment feedback information at low frequencies is thus effectively shifted from analog to digital feedback. This digital feedback is permanently applied, so that the cavity is close to perfect alignment even after a loss of lock, at which point the analog feedback information is lost.

Table 2 summarizes the kind of alignment control applied to each suspended mirror.

\section{Differential Wave-Front Sensing Alignment Loops}

\section{A. Control Filter Design}

The mirrors of the GEO 600 mode cleaners are suspended as double pendulums as shown in the simplified drawing of Fig. 4. Orientation control is applied to the intermediate mass at the locations denoted by arrows. The intermediate mass carries four magnets, on which forces are applied with coils that are rigidly connected to the pendulum's suspension point. (Magnets and coils are not shown for clarity.) The same actuators are used for a separate local control feedback loop, damping the motion on the pendulum resonances.

The mirror is hanging in two steel wire slings and follows the motion of the intermediate mass with a frequency response of $f^{-2}$ above the pendulum resonances, which are around $0.9 \mathrm{~Hz}$ for yaw motions and $1.8 \mathrm{~Hz}$ for pitch motions of the mirror. Owing to the response of the intermediate mass position to the coil current, which also has a $f^{-2}$ response, the overall transfer function from the coil current to the angular motion of the suspended mirror has a $f^{-4}$ response above the pendulum resonances. The optical response of the mode cleaners is frequency independent up to several kilohertz and so does not have to be taken into account here.

For the design of a feedback loop with a bandwidth larger than the pendulum resonances (e.g., with a bandwidth of $6 \mathrm{~Hz}$ ) with this type of actuator, the feedback filter has to provide, in practice, at least $200^{\circ}$ of phase lead around the unity-gain frequency. For this purpose we built analog electronic filters with two sequential biquadratic stages. Care must be taken with the gain distribution of these filters, as a large phase lead comes at the price of a large gain at frequencies higher than the loop bandwidth, which can lead to saturation if no additional low-pass filtering is applied or if the filter input signal is noisy. Table 3 shows the filter parameters for the mirror MMC1b pitch DWS loop of the first mode cleaner.

A second version of the alignment filters follows a completely different design, as it is not aiming for a unity-gain frequency above the pendulum resonances but uses filters with a slope of $f^{-3 / 2}$ below the pendulums' main resonance to have a high loop gain at dc with a unity-gain frequency around $0.2 \mathrm{~Hz}$. As experimental evidence shows, the $0.2-\mathrm{Hz}$ loop is more robust in long-term operation and hence is currently used for both mode cleaners of GEO 600.

\section{B. Results}

The performance of the DWS control system with a bandwidth of around $6 \mathrm{~Hz}$ for four degrees of freedom is shown in Fig. 5.

The time series shows the light power in reflection of the first mode cleaner, detected with D1 in Fig. 2.

Table 2. Alignment Control Signals for All Suspended Mirrors According to Fig. 1

\begin{tabular}{lll}
\hline \multicolumn{1}{c}{ Mirror } & \multicolumn{1}{c}{ Loop Type } & associated sensor \\
\hline BDIMC1 & Spot-position control & PDAMC1 \\
MC1a - MMC1c & DWS control & PDMC1 (DWS signal) \\
MMC1a + MMC1c & Spot-position control & PDMC1 (spot position on D1) \\
MMC1b & DWS control & PDMC1 (DWS signal) \\
BDOMC1 & Spot-position control & PDIMC2 \\
BDIMC2 & Spot-position control & PDIPR \\
MMC2a - MMC2c & DWS control & PDMC2 (DWS signal) \\
MMC2a + MMC2c & Spot-position control & PDMC2 (spot position on D1) \\
MMC2b & DWS control & PDMC2 (DWS signal) \\
BDOMC2 & Spot-position control & Sensor not shown \\
\hline
\end{tabular}




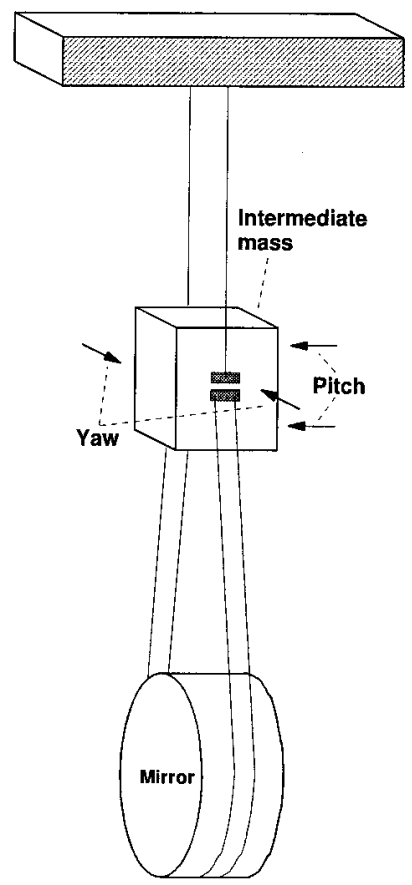

Fig. 4. Simplified double-pendulum suspension. The arrows denote locations where alignment forces are applied by coil and magnet actuators (not shown).

Up to $t=6 s$, the cavity is not in lock, and almost $100 \%$ of the incident light power is reflected. Then the cavity is locked to an intentionally misaligned state with only around $65 \%$ of the possible power entering the cavity. At $t=14 \mathrm{~s}$, the DWS control is switched on for the two pitch degrees of freedom; at $t=19 \mathrm{~s}$, feedback to the two yaw degrees of freedom is switched on, and an optimal cavity alignment is achieved. The remaining reflected power level is due to imperfect annular mode matching at the time this measurement was made.

It can be seen that in this state the power fluctuations are significantly smaller than in the misaligned case, owing to the minimized coupling of beam geometry fluctuations. Finally, the graph shows the switching off in reverse order, with the pendulums relaxing back to their equilibrium positions. (The offset shifting loop was not engaged.)

Figure 6 shows the linear spectral density of the vertical alignment fluctuations of mirror $B$ of the first mode cleaner with alignment feedback (bandwidth is around $6 \mathrm{~Hz}$ ) switched off and on.

This in-loop measurement shows a pitch noise reduction by the alignment system of approximately 20 $\mathrm{dB}$ at $1 \mathrm{~Hz}$ for the first mode cleaner. Note that this

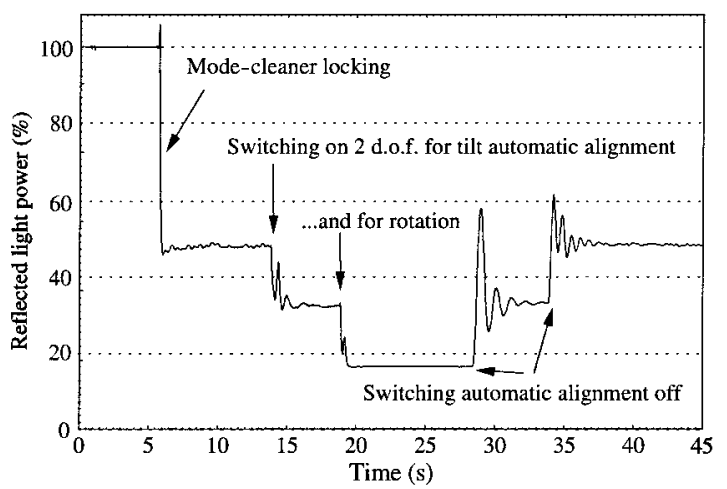

Fig. 5. Time series of the light power reflected from the first GEO 600 mode cleaner; the automatic alignment is switched on and off. At $t=6 s$, the cavity is locked to an intentionally misaligned state with only approximately $65 \%$ of the possible power entering the cavity. At $t=14 \mathrm{~s}$, the DWS control is switched on for the two pitch degrees of freedom; at $t=19 \mathrm{~s}$, feedback to the two yaw degrees of freedom is switched on, and an optimal cavity alignment is achieved. d.o.f., degree of freedom.

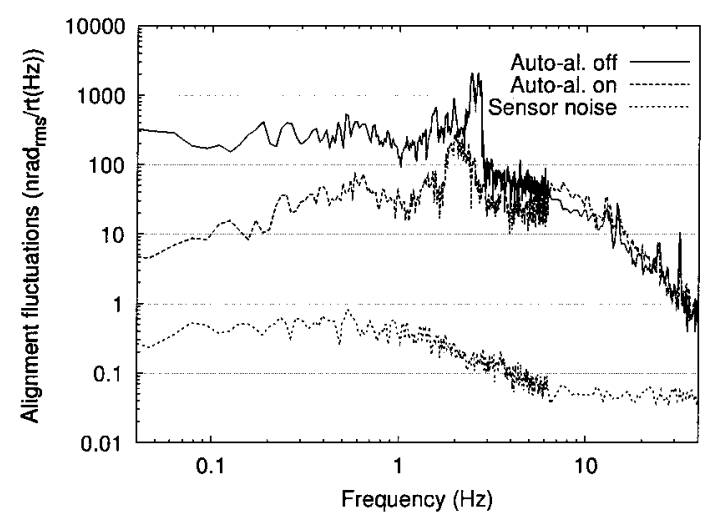

Fig. 6. Spectral density of the DWS signals for mirror MMC1b pitch with alignment feedback switched off and on. The peak around $2 \mathrm{~Hz}$ in the trace without alignment feedback is caused by a suspension mode. The dotted curve shows the sensors noise with no light detected.

performance is achieved with actuation only on the mass above the mirror. Also shown is the sensor noise with no light detected; this is well below the level of residual alignment fluctuations with the automatic alignment switched on.

In addition, we demonstrated a stable long-term operation of an automatic alignment system for the first time. The system described for the two GEO 600 mode cleaners has been in operation since the spring of 2001. No manual realignment of the mode-cleaner mirrors has been required since then

Table 3. Filter Parameters for Mirror MMC1b Pitch DWS Loop with a Bandwidth around $6 \mathrm{~Hz}^{a}$

\begin{tabular}{cccccccc}
\hline Degree of Freedom & Zero $Q$ & Pole $Q$ & Zero $Q$ & Pole $Q$ & Pole $Q$ & Integrator \\
\hline MMC1b pitch & $1.6 \mathrm{~Hz} 2$ & $14 \mathrm{~Hz} 0.7$ & $5 \mathrm{~Hz} 1$ & $20 \mathrm{~Hz} 1$ & $40 \mathrm{~Hz} 2$ & $0.8 \mathrm{~Hz}$ \\
\hline
\end{tabular}

${ }^{a}$ Each entry represents a complex-conjugate pair, except for the last column, which denotes an integrator that is active below the specified frequency. 
(except for a maintenance period in May 2002 that included opening the vacuum system). In contrast, manual alignment corrections were necessary at least every few days before the automatic system was implemented. The automatic alignment system also permits long stretches of continuous locking, with more than $120 \mathrm{~h}$ achieved on a test-run period in August 2002.

\section{Conclusion}

An automatic alignment system, based on differential wave-front sensing, has been implemented for the two suspended, triangular mode-cleaner cavities of GEO 600, by use of three different types of control loop. The important new result of the system is its long-term stability, allowing the mode cleaners to be permanently in use and remain aligned even after losses of lock. The longest continuous lock achieved was more than $120 \mathrm{~h}$. The authors believe that the system is highly satisfactory for use in interferometric gravitational wave detectors with which measurements are intended over long time periods.

The authors thank the Particle Physics and Astronomy Research Council in the U.K., the University of Glasgow, the Bundesministerium für Bildung, Forschung und Technologie, and the State of Lower Saxony in Germany.

\section{References}

1. B. Willke, P. Aufmuth, C. Aulbert, S. Babak, R. Balasubramanian, B. W. Barr, S. Berukoff, S. Bose, G. Cagnoli, M. M. Casey, D. Churches, D. Clubley, C. N. Colacino, D. R. M. Crooks, C. Cutler, K. Danzmann, R. Davies, R. Dupuis, E. Elliffe, C. Fallnich, A. Freise, S. Goßler, A. Grant, H. Grote, G. Heinzel, A. Heptonstall, M. Heurs, M. Hewitson, J. Hough, O. Jennrich, K. Kawabe, K. Kötter, V. Leonhardt, H. Lück, M. Malec, P. W.
McNamara, S. A. McIntosh, K. Mossavi, S. Mohanty, S. Mukherjee, S. Nagano, G. P. Newton, B. J. Owen, D. Palmer, M. A. Papa, M. V. Plissi, V. Quetschke, D. I. Robertson, N. A. Robertson, S. Rowan, A. Rüdiger, B. S. Sathyaprakash, R. Schilling, B. F. Schutz, R. Senior, A. M. Sintes, K. D. Skeldon, P. Sneddon, F. Stief, K. A. Strain, I. Taylor, C. I. Torrie, A. Vecchio, H. Ward, U. Weiland, H. Welling, P. Williams, W. Winkler, G. Woan, and I. Zawischa, "The GEO 600 gravitational wave detector," Class. Quantum Grav. 19, 1377-1387 (2002).

2. E. Morrison, B. J. Meers, D. I. Robertson, and H. Ward, "Automatic alignment of optical interferometers," Appl. Opt. 33, 5041-5049 (1994).

3. E. Morrison, B. J. Meers, D. I. Robertson, and H Ward, "Experimental demonstration of an automatic alignment system for optical interferometers," Appl. Opt. 33, 5037-5040 (1994).

4. G. Heinzel, A. Rüdiger, R. Schilling, K. Strain, W. Winkler, J. Mizuno, and K. Danzmann, "Automatic beam alignment in the Garching 30-m prototype of a laser-interferometric gravitational wave detector," Opt. Commun. 160, 321-334 (1999).

5. R. W. P. Drever, J. L. Hall, F. V. Kowalski, J. Hough, G. M. Ford, A. J. Munley, and H. Ward, "Laser phase and frequency stabilization using an optical resonator," Appl. Phys. B 31, 97-105 (1983).

6. G Heinzel, "Advanced optical techniques for laserinterferometric gravitational-wave detectors," Ph.D. thesis (University of Hannover, Hannover, Germany, 1999). Also available as MPQ Report 243 (Max-Planck-Institut für Quantenoptik, Garching, Germany, 1999) pp. 141-146.

7. S. Goßler, M. M. Casey, A. Freise, A. Grant, H. Grote, G. Heimzel, M. Heurs, M. E. Husman, K. Kötter, V. Leonhardt, H. Lück, M. Malec, K. Mossavi, S. Nagano, P. W. McNamara, M. V. Plissi, V. Quetschke, D. I. Robertson, N. A. Robertson, A. Rüdiger, R. Schilling, K. D. Skeldon, K. A. Strain, C. I. Torrie, H. Ward, U. Weiland, B. Willke, W. Winkler, J. Hough, and K. Danzmann, "Mode-cleaning and injection optics of the gravitational-wave detector GEO 600," Rev. Sci. Instrum. 74, 3787-3795 (2003).

8. M. M. Casey, H. Ward, and D. I. Robertson, "Computer monitoring and control of the GEO 600 gravitational wave detector," Rev. Sci. Instrum. 71, 3910-3917 (2000). 